\title{
Uncertainty and expectancy deviations require cortico-subcortical cooperation
}

Citation for published version (APA):

Mestres-Missé, A., Trampel, R., Turner, R., \& Kotz, S. A. (2017). Uncertainty and expectancy deviations require cortico-subcortical cooperation. Neuroimage, 144(Part A), 23-34.

https://doi.org/10.1016/j.neuroimage.2016.05.069

Document status and date:

Published: 01/01/2017

DOI:

10.1016/j.neuroimage.2016.05.069

Document Version:

Publisher's PDF, also known as Version of record

\section{Please check the document version of this publication:}

- A submitted manuscript is the version of the article upon submission and before peer-review. There can be important differences between the submitted version and the official published version of record.

People interested in the research are advised to contact the author for the final version of the publication, or visit the DOI to the publisher's website.

- The final author version and the galley proof are versions of the publication after peer review.

- The final published version features the final layout of the paper including the volume, issue and page numbers.

Link to publication

\footnotetext{
General rights rights.

- You may freely distribute the URL identifying the publication in the public portal. please follow below link for the End User Agreement:

www.umlib.nl/taverne-license

Take down policy

If you believe that this document breaches copyright please contact us at:

repository@maastrichtuniversity.nl

providing details and we will investigate your claim.
}

Copyright and moral rights for the publications made accessible in the public portal are retained by the authors and/or other copyright owners and it is a condition of accessing publications that users recognise and abide by the legal requirements associated with these

- Users may download and print one copy of any publication from the public portal for the purpose of private study or research.

- You may not further distribute the material or use it for any profit-making activity or commercial gain

If the publication is distributed under the terms of Article $25 \mathrm{fa}$ of the Dutch Copyright Act, indicated by the "Taverne" license above, 


\title{
Uncertainty and expectancy deviations require cortico-subcortical cooperation
}

\author{
Anna Mestres-Missé ${ }^{\mathrm{a}, *, 1}$, Robert Trampel $^{\mathrm{b}}$, Robert Turner ${ }^{\mathrm{b}}$, Sonja A. Kotz ${ }^{\mathrm{b}, \mathrm{c}}$ \\ ${ }^{a}$ School of Psychological Sciences, The University of Manchester, Manchester M13 9PL, United Kingdom \\ ${ }^{\mathrm{b}}$ Max Planck Institute for Human Cognitive and Brain Sciences, Leipzig 04103, Germany

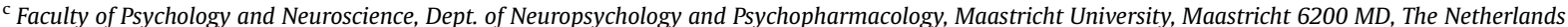

\section{A R T I C L E I N F O}

Article history:

Received 26 October 2015

Received in revised form

29 March 2016

Accepted 27 May 2016

Available online 1 June 2016

Keywords:

Basal ganglia

Thalamus

Red nucleus

Probabilistic inferential learning

Flexible cognitive control

7T-fMRI

\begin{abstract}
A B S T R A C T
In a dynamic and uncertain environment it is beneficial to learn the causal structure of the environment in order to minimize uncertainty. This requires determining estimates of probable outcomes, which will guide expectations about incoming information. One key factor in this learning process is to detect whether an unexpected event constitutes a low probability, but valid outcome, or an outright error. The present 7T-fMRI study investigated the role of subcortical structures in regulating this probabilistic inferential learning process. A new task was designed, in which participants learned to calculate the value, and therefore to anticipate the outcome of different visual sequences. Three types of sequences provided unambiguous, ambiguous, and incongruent contextual evidence and each sequence had two outcomes, which differed in their probability of occurrence. We hypothesized that subcortical regions are necessary when expectations are violated, and that their involvement will depend on the nature of the unexpected event. The results show increased dorsomedial striatal and thalamic activation for less probable sequences; in addition, ambiguous sequences also display larger activation in the red nuclei. Incongruent sequences displayed a pattern of subcortical activation restricted to the dorsolateral and the posterior dorsomedial striatum. These results confirm that different subcortical structures regulate uncertainty and expectancy deviations; this is crucial not only for learning to predict events in the environment, but also for flexible cognitive control in general.
\end{abstract}

(c) 2016 Elsevier Inc. All rights reserved.

\section{Introduction}

Uncertainty and violations of expectations have been suggested to prompt learning (Behrens et al., 2007; O'Reilly, 2013; Yu and Dayan, 2005). Consequently, it has been recently shown that when infants detect discrepancies between actual and expected outcomes, their exploration ('why does the evidence not match my expectations?') and hence learning increases (Stahl and Feigenson, 2015). Determining if an unexpected event is part of a structure or a one-off error is essential to accurately learn about causal structures in the environment so that new incoming information can be faced with a good estimate of what to expect and do. Achieving a well-balanced dynamic between automatic and controlled processing is needed to adjust to changes in the environment, to learn in novel or uncertain situations, to be wary in risky situations, or to

\footnotetext{
* Corresponding author at: School of Psychological Sciences, The University of Manchester, Brunswick Street, Zochonis Building, Manchester M13 9PL, United Kingdom.

E-mail address: anna.mestres-misse@manchester.ac.uk (A. Mestres-Missé).

${ }^{1}$ Data collection was performed while the author was at the Max Planck Institute for Human Cognitive and Brain Sciences, Leipzig, Germany.
}

simply carry on with habits. Cognitive control processes are necessary for successful, flexible behavior as they enable adjustment when habitual behavior is no longer suitable due to environmental uncertainty or changes (for a review see Mushtaq et al., 2011). We have previously demonstrated how the basal ganglia and corticostriatal systems substantially contribute to overriding habitual but currently incompatible behavior, and to engaging the most appropriate response given actual relevant information (MestresMisse et al., 2012, 2014a; cf. den Ouden et al., 2010; Redgrave et al., 1999; Schiffer et al., 2015).

However, before achieving an optimal balance between what is common and thus should be automatic, and what is not, one must first learn to estimate probable outcomes. We have highlighted the relevance of Bayesian statistics, which provide a general and computational principle of learning, adjusting, and updating information to this process (Mestres-Misse et al., 2014a,b). Specifically, given an environment, which provides imprecise, partial, and ambiguous information, the brain combines past experience with observed evidence to estimate probabilistic models, which are used to infer unknown causes and outcomes (Fiser et al., 2010; Penny, 2012; Vilares and Kording, 2011). In a nutshell, we suggest that given actual and previous evidence, the probability of each 
possible outcome is computed; outcomes with a persistent, high probability of occurrence will eventually become dominant and their response automatic. Appropriate responses to lower probability outcomes will only be triggered by either sufficient explicit contextual information, which allows to anticipate and prepare a less common response, or when an inconsistency between the automatic response and the actual evidence is detected. The formation of behavior commences with learning the probabilities of different events in the environment and subsequently creating probability distributions, which update with new evidence. Accordingly, statistical learning efficiency has long been demonstrated in infants (Saffran et al., 1996), and has been ascribed as an essential domain-general learning mechanism of humans and animals (Fiser et al., 2010). Probability distributions allow representing not only the probability of a given outcome, but also its reliability (Behrens et al., 2007; O'Reilly, 2013; Pouget et al., 2013; Yu and Dayan, 2005). The representation of knowledge as probability distributions (Knill and Pouget, 2004), which provide an estimate of uncertainty, allows the brain to perform inferences about the most probable causes and outcomes of events.

We hypothesize that subcortical structures are necessary for modulating information processing and cognitive control, particularly relevant in uncertain environments and when expectations are violated; moreover, their relevance should be especially conspicuous during learning. In the current study we aimed at further demonstrating that a cortico-centric view of executive cognitive functions is limited, and needs to be extended to the essential involvement of subcortical structures, and cortico-subcortical cooperation (for a wider view of this overarching goal, see MestresMisse et al., 2012, 2014a). In order to study these hypotheses, a new task was devised, in which participants learned the probability of particular outcomes as well as to use contextual information to infer these outcomes. Specifically, the task included the following features: 1) unambiguous, ambiguous, and incongruent sequences; these were characterized by the type of contextual information they provided preceding the last element that revealed the outcome. In unambiguous conditions, participants received precise contextual information that they could use to anticipate the upcoming outcome; in ambiguous conditions contextual information did not provide any reliable cues, and in incongruent conditions contextual information was inconsistent with the last element. 2) A dominant and a non-dominant outcome; by manipulating the probability of each outcome, we biased one outcome over another. These two experimental variables correspond to manipulations of the evidence and the priors, respectively. When contextual evidence is ambiguous, priors should have more weight (Fiser et al., 2010; Summerfield and de Lange, 2014); therefore, in the current design, given uncertain contextual information, participants should learn to rely on their knowledge of the probability of each outcome. However, this is not always reliable, and, at times, a conflict between expected (dominant) and actual (non-dominant) outcomes ensues. Furthermore, we compared ambiguous conditions to instances where contextual evidence is precise in anticipating the outcome (either dominant or non-dominant), and to conditions where the contextual evidence is incongruent with either outcome. In the first case, we studied the effects of priors and contextual information on inferring possible outcomes; in the second case, we compared two unexpected conditions, one with a less probable but valid outcome and one that was outright wrong. These manipulations are essential to test our hypothesis that subcortical structures have a decisive and comprehensive role in learning to estimate probable outcomes, and in regulating uncertainty and deviations from expectation; a mechanism, which is indispensable in a dynamic noisy environment, where the estimated expected answer is not always the correct one.

\section{Materials and methods}

\section{Participants}

Twenty-eight right-handed participants without any history of neurological or psychiatric disease participated in the study after giving informed consent. Six participants were excluded from the final sample (one failed to complete the training, one due to bad image quality, and four had less than $65 \%$ correct trials in one or more conditions and/or less than $75 \%$ correct catch trials in the fMRI session). The final sample constituted of twenty-two participants ( $11 \mathrm{fe}-$ males, mean age $25.5 \pm 3.1$, range 20-33). The study was approved by

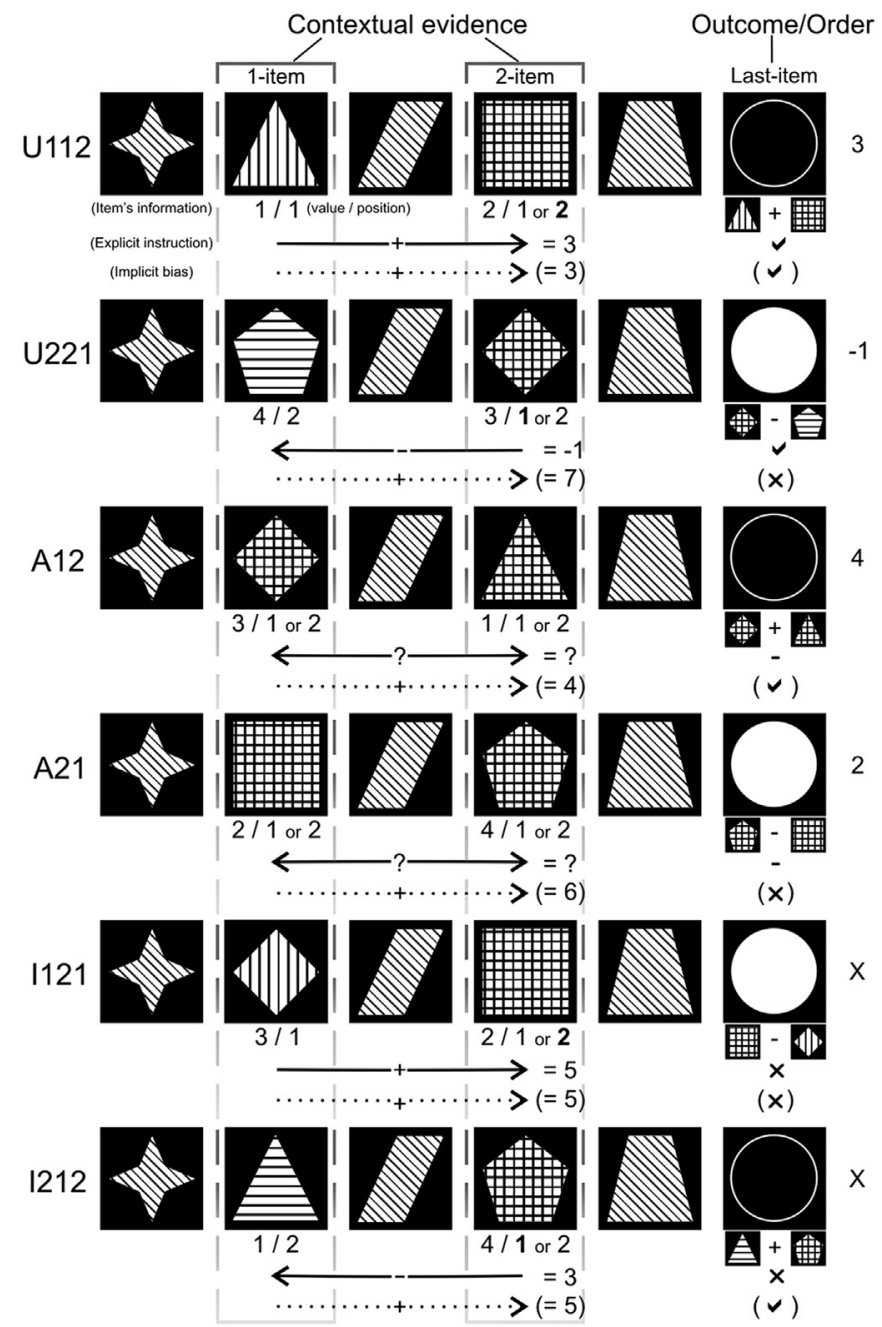

Fig. 1. Stimuli sequences examples for each experimental condition. Participants saw a sequence of six elements. Their task was to calculate the total value of the sequence. To calculate the value of the sequence, they had to either sum 1-item and 2 -item (order 1-2) or to subtract 1-item from 2-item (order 2-1). The last-item indicated whether participants had to perform an addition (black circle, order 1-2, 1 -item +2 -item), or a subtraction (white circle, order 2-1, 2-item - 1-item). Participants could anticipate the outcome/order of the sequence by using the information provided by the items' filling pattern. This information indicated the position of 1-item and 2-item. In unambiguous sequences (U112 and U221), 1-item had an informative filling pattern (vertical stripes indicating 1-item in position 1, thus anticipating 1-item +2 -item; horizontal stripes indicating 1-item in position 2 , thus anticipating 2-item - 1-item). In ambiguous sequences (A12 and A21), the filling pattern of 1-item was ambiguous between position 1 and position 2; thus participants could not anticipate the outcome/order. However, as a bias towards order 1-2 (black circle) was implicitly created (dotted line), in the absence of explicit contextual evidence, participants anticipated order 1-2. This worked well for A12, but for A21 it led to a conflict between expected and actual outcome/order. In incongruent sequences (I121 and I212), the evidence provided by the context was incongruent with the actual outcome/order. 
the research ethics committee of the University of Leipzig.

\section{Experimental paradigm}

Stimuli consisted of sequences of 6 elements (Fig. 1). The first, third, and fifth elements of the sequence were constant across all conditions, and thus will not be further addressed. These elements have been eliminated from future studies, featuring only the three relevant elements described next. The second and fourth elements provided contextual information preceding the final outcome; we will refer to these elements as first (1-item) and second (2-item) items (see boxed elements in Fig. 1). The last element (last-item) revealed the outcome of the sequence. The task of the participants was to calculate the value of each sequence. In order to achieve this, participants were explicitly taught that 1-item and 2-item could correspond to either one of four different geometrical figures, each with an associated value (triangle $=1$; square $=2$; rhombus $=3$; pentagon $=4$ ). Participants were instructed that the last-item of the sequence was either a black or a white circle, and that it indicated what they needed to do with 1-item and 2- item. A black circle meant 'take the first item and add the second' (order 1-2: 1-item +2 -item); a white circle meant 'take the second item and subtract the first' (order 2-1: 2-item - 1-item). In addition, participants were explicitly taught that the filling pattern of 1 -item and 2 -item provided information regarding the position the item could take in the mathematical equation (i.e. position $1 \pm$ position 2 ). Vertical stripes meant that the item could only be in position 1 , horizontal stripes meant that the item could only be in position 2 , and a crossed pattern indicated that the item could be in either position. 1-item could have one of the three filling patterns, while 2-item always had a crossed pattern. The filling pattern of the item thus was a contextual cue that provided information for the participants to anticipate what to do with the two items, and thus the value of the sequence, before the last item. In summary, 1-item with vertical stripes cued order 1-2 [1-item (position 1) + 2-item (position 2)], while 1-item with horizontal stripes cued order 2-1 [2item (position 1) - 1-item (position 2)]. For instance, the first example in Fig. 1 is an unambiguous sequence (U112), as explicit contextual information is provided by the filling pattern of 1-item. 1 -item is a vertically striped triangle (value $=1$ ), the filling pattern denotes that this item can only be in position 1 (U112). At this point, participants can anticipate a black circle (order 1-2, triangle +2 item), and thus, they can start calculating the value of the sequence (instead of having to wait for the circle to tell them what to do). 2 -item is a crossed square (value $=2$ ), that is, it can be in any position, but because position 1 is already taken by 1 -item (triangle), the square (2-item) must be in position 2 (i.e. triangle + square). The last-item is a black circle, which is congruent with what the item patterns indicated and the participants expected (order 1-2; U112). The value of this sequence is 3 .

The experiment included six critical conditions: three different types of sequences (unambiguous, ambiguous, incongruent) and two orders (1-2, 2-1) (Fig. 1). For unambiguous conditions (Shannon, bit entropy $=0$ ), 1 -item had an explicit filling pattern (vertical: position 1 ; horizontal: position 2 ), which was congruent with the final element of the sequence (black circle: order 1-2; white circle: order 2-1), hence, U112 and U221 sequences. For ambiguous conditions (Shannon, bit entropy =1), 1-item had an uncertain filling pattern (crossed); thus, participants could not know what to do with the items until the last-item, hence, A12 and A21 sequences. For incongruent conditions, 1-item had an explicit pattern, but this was not congruent with the last-item, hence, I121 and I212. In addition, two filler conditions were added (F12 and F21, not shown on Fig. 1). These were identical to A12 and A21 conditions with the exception that the filling pattern of the 5th element (trapezoid) was not the same as the constant one (remember that the 1st, 3rd and 5th elements were constant with a common pattern). Participants were taught that this inconsistency made the sequence "incorrect".

A critically important feature of the current research was the creation of a bias towards a particular sort of sequence (i.e. order $1-2$ ), so that even in the case of ambiguous contextual information, participants would learn a preferred, dominant process, and a less common, non-dominant one. Therefore, conditions were designed so that the non-dominant ambiguous condition would require the highest processing demands, as in the instance of an ambiguous sequence, participants would anticipate a dominant order (1-2), and thus their expectation of this order would clash with the actual outcome (last element, 2-1). In the case of unambiguous conditions, even though the context provided cues about the outcome, the existence of a bias, would render the non-dominant unambiguous condition less probable, and consequently more demanding than the dominant one. In order to achieve the bias, as well as ensuring participants' automatic calculation of the sequences, participants underwent three consecutive training sessions before the MRI session, which took place on the fourth day after three days consecutive training. During the three training sessions, the ratio of order 1-2/2-1 was 75:25, thus creating a bias towards order 1-2. During the MRI session the ratio was 50:50. Participants were explicitly informed of all rules, except the manipulation of the order 1-2/2-1 ratio; thus, the bias was created implicitly.

In sum, the current experiment involved 4 consecutive sessions (3 training, 1 scanning). The aim of the training was two-fold; on one hand, it habituated participants to calculate the sequences value, and, on the other hand, it implicitly created a processing bias towards order 1-2. These two features were crucial because during the fMRI session: 1) participants were asked to decide if a sequence was correct or incorrect (see below for detailed information), thus we needed to ensure that the calculation was done automatically, and 2) stimuli presentation was balanced, therefore, we had to ensure a previously created processing bias.

For details on the training see Inline Supplementary Methods.

The fMRI session differed from the training sessions in the following way: 1) all conditions had the same number of trials, 2) participants made a correctness judgment, 3) catch trials were introduced, and 4) no feedback was provided. Prior to the scanning session, participants were instructed that their task was to judge whether a sequence was correct or incorrect. They were also told that sometimes after the decision a number and a question mark would appear; in these cases they had to report whether this was or was not the value of the sequence. Stimuli consisted of $24 \mathrm{U} 112$, 24 U221, 24 A12, 24 A21, 24 I121, 24 I212, 24 F12 and 24 F21 sequences. These were divided into 4 runs, each containing 6 unique sequences per condition.

Each run started with two baseline images ( $4 \mathrm{~s}$ ). Each trial began with a fixation cross lasting $500 \mathrm{~ms}$, then sequences were presented element by element in the center of the screen (duration $=500 \mathrm{~ms}$, $\mathrm{SOA}=700 \mathrm{~ms}$ ). After $500 \mathrm{~ms}$, a prompt was presented for $4 \mathrm{~s}$ (or until button-press) asking participants to indicate whether the sequence was correct or incorrect by pressing one of two buttons (the responding-hand was counterbalanced across participants). In the following, on half of the correct trials (unambiguous and ambiguous conditions), a number and a question mark were presented for $4 \mathrm{~s}$ (or until button-press) and participants had to indicate by pressing one of two buttons whether this was the value of the sequence. The screen remained dark for a variable 1 - to 6 -second interval. Subsequently, the next sequence was presented in the same fashion. Each run lasted approximately $11 \mathrm{~min}$. The order of the experimental conditions within an experimental run was pseudo-randomized with the restriction that the same condition could not occur more than two times in a row. Stimulus presentation was controlled by Presentation software (http://www.neurobs.com/) and synchronized with the MRI data 
acquisition with an accuracy of $1 \mathrm{~ms}$. Stimuli were presented on a black background and projected onto a screen and could be viewed by the participant through a mirror system mounted onto the head-coil.

\section{MRI data acquisition}

Images were acquired on a 7T whole-body MR scanner (MAGNETOM 7T, Siemens Healthcare, Erlangen, Germany) using a 24channel phased array head coil (NOVA Medical Inc., Wilmington, MA, USA). T2*-weighted functional magnetic resonance images were obtained (330 scans per run) using an axially oriented echoplanar imaging (EPI) sequence covering a partial volume that included subcortical structures $(\mathrm{TR}=2000 \mathrm{~ms}$; $\mathrm{TE}=18 \mathrm{~ms}$; flip angle $=75^{\circ} ; 44$ slices; $1.5 \mathrm{~mm}$ thickness; no gap; matrix size: $128 \times 128$; field of view: $192 \times 192$; resolution: $1.5 \mathrm{~mm}$ isotropic voxels; $\quad$ bandwidth $=1776 \mathrm{~Hz} / \mathrm{Px} ; \quad$ GRAPPA reconstruction; iPAT $=3$ ). Fieldmap data were acquired using two gradient-echoes with time difference of $1.02 \mathrm{~ms}$ ( TR $=1000 \mathrm{~ms}$; TE $=6.00 / 7.02 \mathrm{~ms}$; flip angle $=75^{\circ} ; 33$ slices; $2 \mathrm{~mm}$ isotropic voxels). For anatomical reference whole-brain $\mathrm{T} 1$ weighted structural data were acquired with an MP2RAGE (Marques et al., 2010) sequence (TR = $5000 \mathrm{~ms}$; $\mathrm{TE}=2.45 \mathrm{~ms} ; \mathrm{TI}_{1 / 2}=900 / 2750 \mathrm{~ms} ;$ flip angle $_{1 / 2}=5 / 3^{\circ} ; 0.7 \mathrm{~mm}$ isotropic voxels; GRAPPA reconstruction; iPAT $=2$ ).

As the exact functional identification of subcortical structures requires a very high spatial resolution, a sufficient SNR is necessary. Therefore, we used a 7T-MR scanner for our fMRI experiment, as this field strength offers more than twice the SNR of more conventional 3T scanners (Edelstein et al., 1986). However, the prolonged EPI readout time of an imaging matrix encoding $1.5 \mathrm{~mm}$ isotropic voxels requires a decreased number of acquired k-space lines, which can be achieved using parallel imaging. This technique has been used in many fMRI studies, especially at high-field strengths such as 7T (van der Zwaag et al., 2015), and proved to deliver high SNR at high spatial resolution. This is also true in subcortical areas, where the well-known g-factor penalty may lead to a decreased SNR compared to areas closer to the coil elements (Pruessmann et al., 1999). Furthermore, 7T-MRI was shown to identify smallest brain structures both structurally and functionally (van der Zwaag et al., 2015). Even a tiny nucleus such as the habenula was localized in vivo using ultra-high field MRI (Strotmann et al., 2014). Therefore, the structures under investigation in this study, such as subcortical structures and nuclei are manageable targets to image given the use of highly developed scanner and coil hardware.

\section{MRI data analysis}

Pre-processing of functional MRI data, including fieldmap correction, slice timing, realignment, coregistration, normalization (DARTEL) on the basis of the segmented high-resolution structural image, and smoothing with a 3-mm full-width half-maximum (FWHM) isotropic Gaussian kernel, was performed using SPM12 (http://www.fil.ion.ucl. ac.uk/spm). The statistical evaluation was based on a least-square estimation using a general linear model approach (Friston et al., 1995). The event-related design matrix included all conditions of interest, that is, U112, U221, A12, A21, I121 and I212, and three conditions of no interest, two fillers (F12 and F21) and incorrectly responded trials. Only trials in which participants responded correctly were analysed. Onsets were set at the final element (circle) of the sequence. The data were high-pass filtered (to a maximum of $1 / 128 \mathrm{~Hz}$ ), and serial autocorrelations were estimated using an autoregressive model (AR (1) model). Task-correlated motion effects were minimized by including the estimated movement parameters. Contrast images were calculated for each participant. The resulting contrast images were submitted to the second-level analysis using two 2-way repeated measures ANOVAs, one with the factors 'type of sequence' (ambiguous (A), unambiguous $(\mathrm{U})$ ) and 'order' (1-2, 2-1) and the other one with the factors 'type of sequence' (ambiguous (A), incongruent (I)) and 'order'
(1-2, 2-1), both with correction for non-sphericity. These analyses were performed separately because: 1) for incongruent sequences order was not a straightforward manipulation, thus, the main effect of order could only be fully assessed in the correct conditions (ambiguous and unambiguous), and 2) the principal goal of the study was the comparison of ambiguous sequences to unambiguous and incongruent sequences. In the case of the ambiguous/incongruent statistical model, the interaction specifically tested whether the contrast $2-1>1-2$ was greater in ambiguous than incongruent conditions. In order to follow-up interactions, and to further study the main effect of order, we performed a t-test assessing the differences between A21 and A12 (exclusively masked by the contrast U221 vs U112).

A whole-brain multiple regression analysis was also performed to test correlation between functional data and the difference between response times in ambiguous non-dominant (A21) minus unambiguous non-dominant (U221) conditions. Furthermore, functional connectivity analyses assessing higher inter-regional coupling with the below defined ROIs during the A21 condition were performed with gPPI (http://www.nitrc.org/projects/gppi) (McLaren et al., 2012) using as seed regions $1 \mathrm{~mm}$ radius spheres around the peak coordinates of the left and right centromedianparafascicular (CM-Pf) complex, and the left and right anterior and posterior dorsomedial striatum (DMS). The first level gPPI results were taken to a second level random effects analysis using a onesample t-test to assess group effects. These seed regions were chosen based on our previous research and hypotheses, as well as the established anatomically connections between the CM-Pf complex and the basal ganglia (Parent and Hazrati, 1995).

Unless otherwise noted, all statistics are FWE corrected at the cluster level for multiple comparisons at $\mathrm{p}<.05$ with a height threshold of $\mathrm{p}<.001$ uncorrected. Maxima and all coordinates are reported in MNI coordinates as used by SPM. All data were superimposed on the mean anatomical image formed by averaging all 22 participants' T1 weighted structural data mapped into normalized MNI space. Anatomical assessment of the significantly activated regions was performed following the Mai atlas (Mai and Paxinos, 2008) and the Morel atlas of the thalamus and basal ganglia (Morel, 2007).

Maps of parameter estimates ( $\beta$ values) were computed from the generalized linear model to assess the magnitude of activation during each condition. The mean parameter estimate of each regressor was then calculated at the cluster activation maximum for each participant and region. Since our a priori hypothesis and evidence (Mestres-Misse et al., 2012, 2014a) of differential DMS regions involvement, these parameter estimates were used as dependent variables in a 4-way mixed ANOVA with the repeated factors 'type of sequence' (ambiguous, unambiguous, incongruent) and 'order' (1-2, 2-1), and the between-subjects factors 'laterality' (left, right) and 'anteriority' (anterior, posterior). This analysis was followed by two 3-way mixed ANOVAs with the repeated factors 'type of sequence' (ambiguous vs. unambiguous; ambiguous vs. incongruent) and 'order' (1-2, 2-1), and the between-subjects factor 'anteriority' (anterior, posterior), performed separately for the left and right DMS. The focus of these analyses was to assess interaction effects between the different DMS subregions and experimental conditions; no analysis was performed on a singular region, thus avoiding circularity.

\section{Results}

\section{Behavioral performance}

The analysis of the training revealed that participants found it more difficult to process the ambiguous sequences compared to the other two sequences, especially in the case of the non-dominant order 2-1 (Table 1, and Inline Supplementary Results and Inline Supplementary Table S1). Although initially participants 
Table 1

Behavioral performance.

\begin{tabular}{|c|c|c|c|c|c|c|c|c|c|c|c|c|}
\hline & U112 & & U221 & & A12 & & A21 & & I121 & & I 212 & \\
\hline & RT & $\% \mathrm{C}$ & RT & $\% \mathrm{C}$ & $\mathrm{RT}$ & $\% \mathrm{C}$ & RT & $\% \mathrm{C}$ & RT & $\% \mathrm{C}$ & $\mathrm{RT}$ & $\% \mathrm{C}$ \\
\hline Day 1 & $433.8(152.6)$ & $97.7(2.4)$ & 605.7 (305.7) & $80.3(26.8)$ & 454.9 (179.4) & $98.3(1.8)$ & $658.9(344.9)$ & 83.7 (10.9) & - & - & - & - \\
\hline Day 2 & $836.2(262)$ & 90.5 (10.9) & $1345(584)$ & $75.4(13.8)$ & $688.8(213)$ & $95.6(7.1)$ & $1654(453)$ & $75.7(13.8)$ & $\begin{array}{l}711.3 \\
(290.8)\end{array}$ & $\begin{array}{l}87.5 \\
(13.9)\end{array}$ & $\begin{array}{l}718.9 \\
(312.2)\end{array}$ & $\begin{array}{l}86.4 \\
(17.2)\end{array}$ \\
\hline Day 3 & $684.2(244.2)$ & $94.3(5.4)$ & $1049.3(602.2)$ & $91.3(12.7)$ & $587.9(212.8)$ & $97.5(3.7)$ & $1327(365.8)$ & $79.2(11.4)$ & $\begin{array}{l}547.3 \\
(224.6)\end{array}$ & $\begin{array}{l}95.7 \\
(5.4)\end{array}$ & $\begin{array}{l}564.5 \\
(225.8)\end{array}$ & $\begin{array}{l}91.7 \\
(13.8)\end{array}$ \\
\hline Day 4 & $524.9(234.2)$ & 99.4 (1.9) & $555.4(249.6)$ & $97.3(4.4)$ & $461.4(168.5)$ & $99(1.8)$ & $666.1(178.5)$ & $97.9(3.1)$ & $\begin{array}{l}481.1 \\
(205.9)\end{array}$ & $\begin{array}{l}94.3 \\
(4.4)\end{array}$ & $\begin{array}{l}478.5 \\
(208.3)\end{array}$ & $93.6(8)$ \\
\hline
\end{tabular}

Response times (ms) and percentage of correct responses for the different sequence conditions. Standard deviation in parentheses.

were less accurate in processing all non-dominant (order 2-1) sequences, by the third training day this effect had disappeared for the unambiguous sequences, but remained for the ambiguous sequences. Thus participants had indeed learned to use explicit information provided by the context to calculate the value of the sequence. Although they demonstrated great accuracy for unambiguous sequences, participants were still slower to process the non-dominant order 2-1 than the dominant order 1-2. Additionally, as expected, the factor order was not relevant for incongruent sequences. Thus, the training was successful, in that, participants were able to anticipate the value of a sequence before the last-item, when unambiguous contextual cues were provided, and developed a processing bias towards the order 1-2. As a result of this bias, A21 sequences conflicted with probabilistic expectations as in the absence of precise contextual evidence, participants learned to anticipate the dominant order 1-2.

The analysis of the training revealed that participants found it more difficult to process the ambiguous sequences compared to the other two sequences, especially in the case of the non-dominant order 2-1 (Table 1, and Inline Supplementary Results and Inline Supplementary Table S1). Although initially participants were less accurate in processing all non-dominant (order 2-1) sequences, by the third training day this effect had disappeared for the unambiguous sequences, but remained for the ambiguous sequences. Thus participants had indeed learned to use explicit information provided by the context to calculate the value of the sequence. Although they demonstrated great accuracy for unambiguous sequences, participants were still slower to process the non-dominant order 2-1 than the dominant order 1-2. Additionally, as expected, the factor order was not relevant for incongruent sequences. Thus, the training was successful, in that, participants were able to anticipate the value of a sequence before the last-item, when unambiguous contextual cues were provided, and developed a processing bias towards the order 1-2. As a result of this bias, A21 sequences conflicted with probabilistic expectations as in the absence of precise contextual evidence, participants learned to anticipate the dominant order 1-2.

Inline Supplementary Table S1 can be found online at http://dx. doi.org/10.1016/j.neuroimage.2016.05.069.

For the fMRI analysis, response times and accuracy scores were used as dependent variables in a two-way repeated-measures ANOVA with the factors 'type of sequence' (unambiguous, ambiguous, incongruent) and 'order' (1-2, 2-1).

The analysis of the response times showed a significant main effect of order $\left(\mathrm{F}_{1,21}=12.56, \mathrm{p}=.002, \mathrm{y}_{\mathrm{p}}{ }^{2}=.38\right)$ and a significant interaction between type of sequence and order $\left(F_{2,42}=18.79, p<.001\right.$, $\left.\mathrm{y}_{\mathrm{p}}{ }^{2}=.47\right)$ (Table 1$)$. The main effect of type of sequence was not significant $\left(\mathrm{F}_{2,42}=2.59, \mathrm{p}=.086, \mathrm{\eta}_{\mathrm{p}}{ }^{2}=.11\right)$. Slower response times for order 2-1 compared to order 1-2 were observed only for ambiguous sequences $\left(\mathrm{t}_{21}=6.22, \mathrm{p}<.001\right)$ (U112 vs. U221: $\mathrm{t}<1$; I121 vs. $\mathrm{I} 212=\mathrm{t}<1)$. A two-way repeated-measures ANOVA restricted to unambiguous and ambiguous conditions revealed a main effect of order $\left(\mathrm{F}_{1,21}=14.73, \mathrm{p}=.001, \mathrm{y}_{\mathrm{p}}{ }^{2}=.41\right)$ and a significant interaction between order and type of sequence $\left(\mathrm{F}_{1,21}=43.94, \mathrm{p}<.001\right.$, $\left.\mathrm{y}_{\mathrm{p}}{ }^{2}=.68\right)$. No significant main effect of type of sequence was found $(\mathrm{F}<1)$. Shorter response times were observed for unambiguous 2-1 sequences compared to ambiguous $2-1$ sequences $\left(t_{21}=-2.88\right.$, $\mathrm{p}=.009$ ), but no differences were found between unambiguous $1-2$ sequences and ambiguous $1-2$ sequences $\left(t_{21}=1.83, \mathrm{p}=.081\right)$. A two-way repeated-measures ANOVA including the factors type of sequence' (ambiguous, incongruent) and 'order' (1-2, 2-1) showed significant main effects of type of sequence $\left(\mathrm{F}_{1,21}=6.15, \mathrm{p}=.022, \mathrm{n}_{\mathrm{p}}^{2}\right.$ $=.23)$, order $\left(\mathrm{F}_{1,21}=27.27, \mathrm{p}<.001, \mathrm{y}_{\mathrm{p}}^{2}=.57\right)$ as well as a significant interaction between these factors $\left(\mathrm{F}_{1,21}=24.75, \mathrm{p}<.001\right.$, $\left.\mathrm{\eta}_{\mathrm{p}}{ }^{2}=.54\right)$. In general, incongruent sequences were responded to faster than ambiguous sequences.

For accuracy, there was a significant main effect of type of sequence $\left(\mathrm{F}_{1.306,27.429}=10.34, \mathrm{p}=.002, \mathrm{y}_{\mathrm{p}}{ }^{2}=.33\right)$ and a marginally significant effect of order $\left(\mathrm{F}_{1,21}=3.66, \mathrm{p}=.069, \mathrm{y}_{\mathrm{p}}{ }^{2}=.15\right)$ (Table 1). No significant interaction between these factors was found $(\mathrm{F}<1)$. Bonferroni corrected pairwise comparisons revealed a significantly lower percentage of correct responses for incongruent sequences than unambiguous $(\mathrm{p}=.016)$ and ambiguous $(\mathrm{p}=.003)$ sequences. A two-way repeated-measures ANOVA for unambiguous and ambiguous conditions revealed only a main effect of order $\left(\mathrm{F}_{1,21}=6.92, \mathrm{p}=.016, \mathrm{y}_{\mathrm{p}}{ }^{2}=.25\right)$. Higher percentage of correct answers were found for order 1-2 sequences compared to order 2-1 sequences. There were no accuracy scores differences between incongruent sequences (I121 vs. I212: $\mathrm{t}<1$ ).

The analysis of the behavioral data revealed that the processing bias created during the training persisted in the fMRI session even with equalized conditions. Non-dominant (order 2-1) sequences displayed a slightly lower percentage of correct responses than dominant (order 1-2) sequences. Furthermore, only ambiguous sequences showed slower response times for order 2-1 compared to order 1-2 sequences. Consequently, the ambiguous order 2-1 condition appears to be the most cognitively demanding condition.

\section{Functional imaging}

fMRI data analysis comparing ambiguous and unambiguous conditions revealed significantly larger activations in the right red nucleus (RN), left angular gyrus, and medial and rostral frontal cortex for ambiguous compared to unambiguous sequences (Figs. 2 and 3A, Inline Supplementary Table S2). Unambiguous sequences compared to ambiguous sequences showed significantly larger activation mainly in parietal regions (Inline Supplementary Table S2). The contrast between order 2-1 minus order 1-2 sequences yielded significantly larger subcortical activation in bilateral anterior and posterior DMS, and the left ventral anterior thalamic nucleus (VA) (Fig. 2, Inline Supplementary Table S2). Furthermore, larger significant cortical activation was found in visual processing regions; parietal cortices, including bilateral intraparietal sulcus, superior parietal lobule and precuneus; left posterior middle 


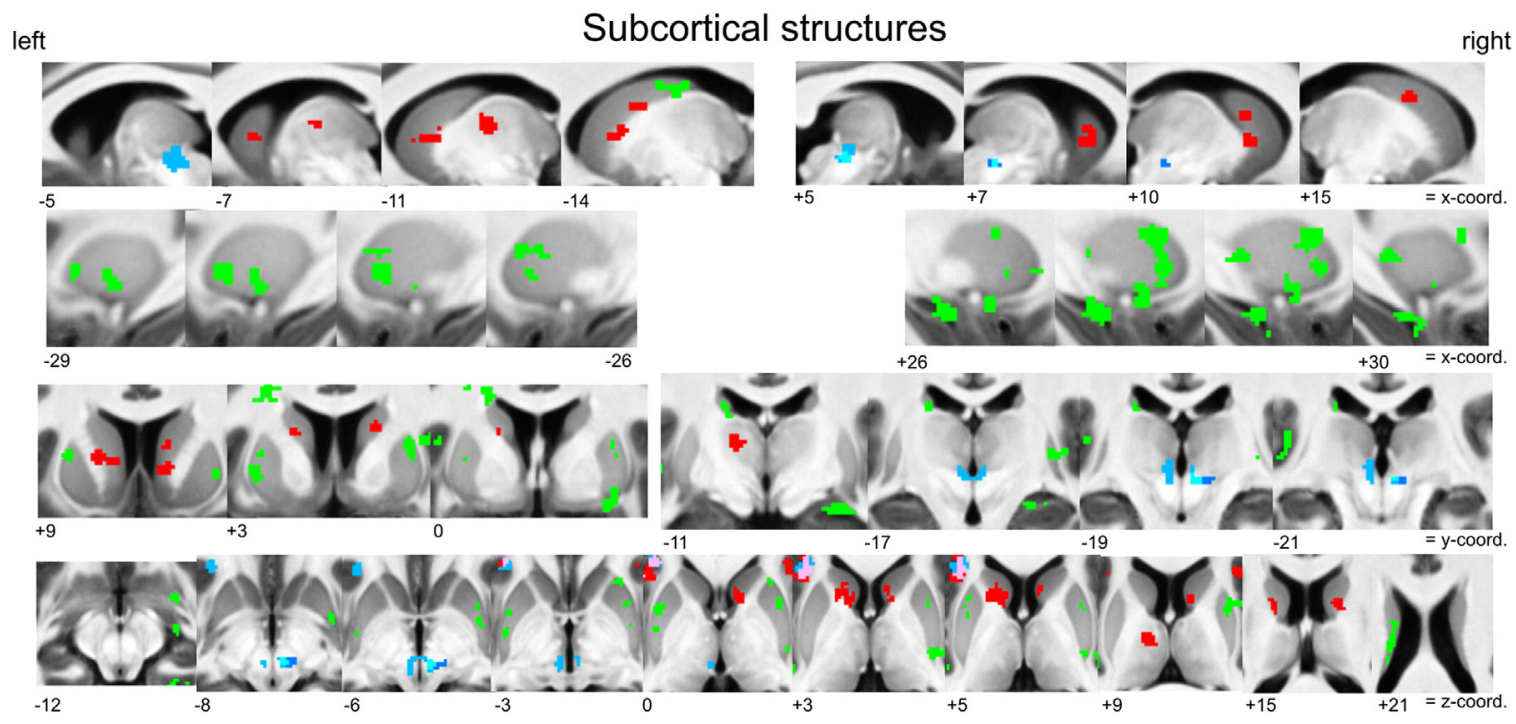

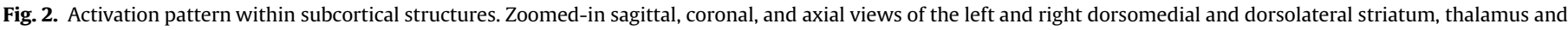

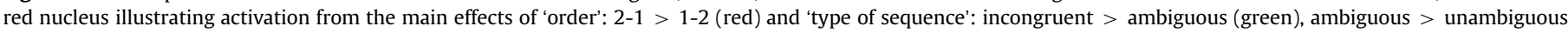
(dark blue), ambiguous > incongruent (blue), overlapping activation from ambiguous $>$ unambiguous and ambiguous $>$ incongruent (cyan).

temporal gyrus; as well as left precentral sulcus, left inferior frontal gyrus (opercular part), left anterior and posterior dorsolateral prefrontal cortex, bilateral anterior insula and dorsal anterior cingulate cortex (Fig. 3B, Inline Supplementary Table S2). For a list of significant activations in the reverse contrast (order 1-2 minus order 2-1) see Inline Supplementary Table S2. A significant interaction between type of sequence and order was found in the right anterior insula (Fig. 3C, Inline Supplementary Table S2).

fMRI data analysis comparing ambiguous and unambiguous conditions revealed significantly larger activations in the right red nucleus (RN), left angular gyrus, and medial and rostral frontal cortex for ambiguous compared to unambiguous sequences (Figs. 2 and 3A, Inline Supplementary Table S2). Unambiguous sequences compared to ambiguous sequences showed significantly larger activation mainly in parietal regions (Inline Supplementary Table S2). The contrast between order 2-1 minus order 1-2 sequences yielded significantly larger subcortical activation in bilateral anterior and posterior DMS, and the left ventral anterior thalamic nucleus (VA) (Fig. 2, Inline Supplementary Table S2). Furthermore, larger significant cortical activation was found in visual processing regions; parietal cortices, including bilateral intraparietal sulcus, superior parietal lobule and precuneus; left posterior middle temporal gyrus; as well as left precentral sulcus, left inferior frontal gyrus (opercular part), left anterior and posterior dorsolateral prefrontal cortex, bilateral anterior insula and dorsal anterior cingulate cortex (Fig. 3B, Inline Supplementary Table S2). For a list of significant activations in the reverse contrast (order 1-2 minus order 2-1) see Inline Supplementary Table S2. A significant interaction between type of sequence and order was found in the right anterior insula (Fig. 3C, Inline Supplementary Table S2).

Inline Supplementary Table S2 can be found online at http://dx. doi.org/10.1016/j.neuroimage.2016.05.069.

The analysis comparing ambiguous and incongruent sequences revealed that ambiguous sequences gave significantly larger bilateral activation in the CM-Pf thalamic nuclear complex and RN (Fig. 2, Inline Supplementary Table S3) and in the left anterior insula (Fig. 3D, Inline Supplementary Table S3). The comparison between incongruent minus ambiguous sequences showed significantly larger subcortical activation for incongruent sequences in bilateral anterior and posterior dorsolateral striatum (DLS), ventral DLS, the tail of the caudate, as well as in the most posterior DMS (body of the caudate, more caudal and dorsal than the activation found in the previous analysis, Fig. 2 top panel) (Fig. 2, Inline Supplementary Table S3). For a list of significantly larger cortical activations for incongruent compared to ambiguous sequences see Inline Supplementary Table S3. A significant interaction between type of sequence and order was found in bilateral primary and secondary visual cortices, left precentral gyrus, and right anterior insula (Fig. 3E, Inline Supplementary Table S3).

The analysis comparing ambiguous and incongruent sequences revealed that ambiguous sequences gave significantly larger bilateral activation in the CM-Pf thalamic nuclear complex and RN (Fig. 2, Inline Supplementary Table S3) and in the left anterior insula (Fig. 3D, Inline Supplementary Table S3). The comparison between incongruent minus ambiguous sequences showed significantly larger subcortical activation for incongruent sequences in bilateral anterior and posterior dorsolateral striatum (DLS), ventral DLS, the tail of the caudate, as well as in the most posterior DMS (body of the caudate, more caudal and dorsal than the activation found in the previous analysis, Fig. 2 top panel) (Fig. 2, Inline Supplementary Table S3). For a list of significantly larger cortical activations for incongruent compared to ambiguous sequences see Inline Supplementary Table S3. A significant interaction between type of sequence and order was found in bilateral primary and secondary visual cortices, left precentral gyrus, and right anterior insula (Fig. 3E, Inline Supplementary Table S3).

Inline Supplementary Table S3 can be found online at http://dx. doi.org/10.1016/j.neuroimage.2016.05.069.

The observed interaction effects were caused by a larger order 2-1 vs order 1-2 increase of activation for ambiguous (A21 > A12) than for the other conditions (Inline Supplementary Fig. S3 and Inline Supplementary Table S4). Furthermore, A21 > A12 accounted for the bulk of the activation corresponding to the main effect of order (Inline Supplementary Fig. S3 and Inline Supplementary Table S4). Only bilateral visual cortices (height threshold: $\mathrm{p}=.001 ;$ peak voxel: $-8, \quad-68,9 ; \mathrm{t}=11.49 ; \mathrm{kE}=2364$; pFWE $<.001)$ and the left precentral sulcus/inferior frontal gyrus (opercular part) (height threshold: $\mathrm{p}=.001$; peak voxel: $-53,8$, $12 ; \mathrm{t}=6.03 ; \mathrm{kE}=121 ; \mathrm{pFWE}=.002$ ) showed a significant correspondence between ambiguous and unambiguous conditions when A21 > A12 was inclusively masked with U221 > U112 (Inline Supplementary Fig. S3 and Inline Supplementary Table S4).

The observed interaction effects were caused by a larger order 2-1 vs order 1-2 increase of activation for ambiguous (A21 > A12) 
a. Ambiguous $>$ Unambiguous
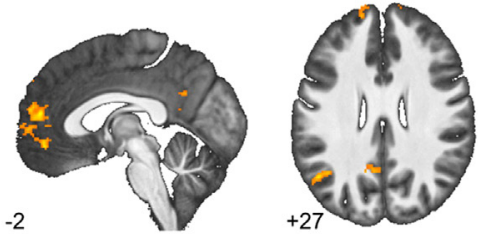

3.5

$$
4.5
$$

$$
5.5
$$

c. Interaction

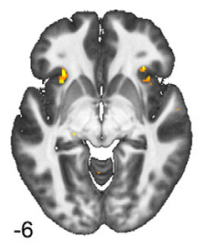

20
16

14

b. Order 2-1 > Order 1-2

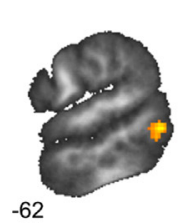

$-62$

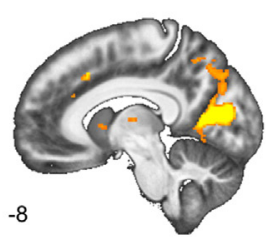

$-48$
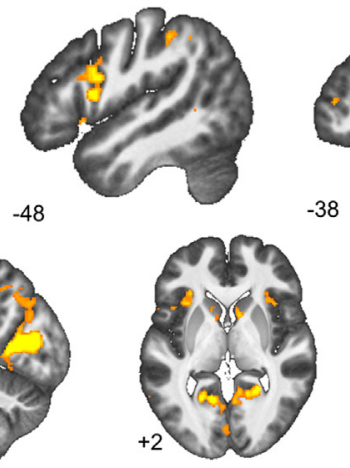
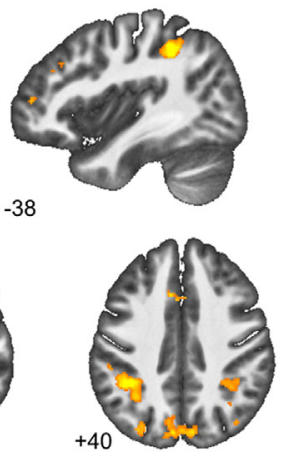

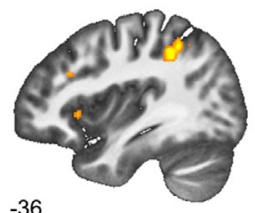

$-36$

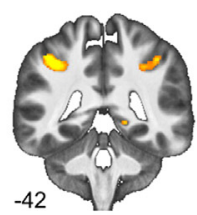

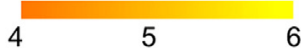

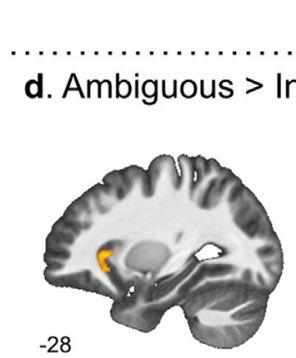
6

e. Interaction

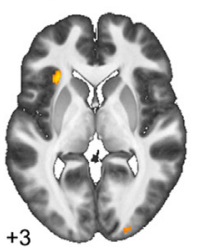

3.5
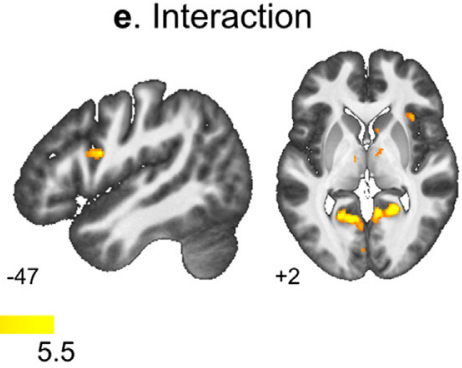

4.5

5.5

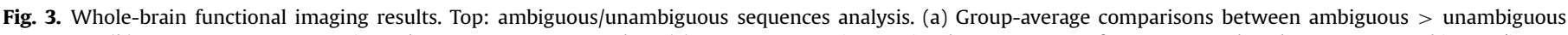

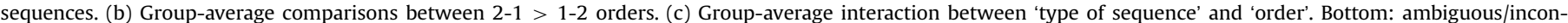

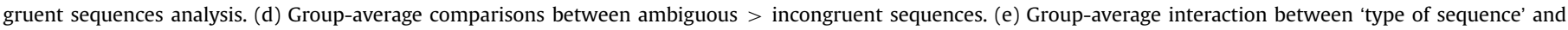
'order'.

than for the other conditions (Inline Supplementary Fig. S3 and Inline Supplementary Table S4). Furthermore, A21 > A12 accounted for the bulk of the activation corresponding to the main effect of order (Inline Supplementary Fig. S3 and Inline Supplementary Table S4). Only bilateral visual cortices (height threshold: $\mathrm{p}=.001 ;$ peak voxel: $-8, \quad-68,9 ; \mathrm{t}=11.49 ; \mathrm{kE}=2364$; pFWE $<.001$ ) and the left precentral sulcus/inferior frontal gyrus (opercular part) (height threshold: $\mathrm{p}=.001$; peak voxel: $-53,8$, $12 ; \mathrm{t}=6.03 ; \mathrm{kE}=121 ; \mathrm{pFWE}=.002$ ) showed a significant correspondence between ambiguous and unambiguous conditions when A21 > A12 was inclusively masked with U221 > U112 (Inline Supplementary Fig. S3 and Inline Supplementary Table S4).

Inline Supplementary Fig. S3 and Table S4 can be found online at http://dx.doi.org/10.1016/j.neuroimage.2016.05.069.

\section{Parameter estimates analysis}

A more exhaustive analysis of striatal activations was performed on the different DMS regions (Fig. 4). This analysis revealed significant main effects of order $\left(F_{1,84}=98.99\right.$, $p<.001$, $\left.\mathrm{\eta}_{\mathrm{p}}{ }^{2}=.54\right)$ and laterality $\left(\mathrm{F}_{1,84}=4.62, \mathrm{p}=.034, \mathrm{\eta}_{\mathrm{p}}{ }^{2}=.05\right)$ as well as significant interactions between type of sequence and anteriority $\left(\mathrm{F}_{2,168}=11.04, \mathrm{p}<.001, \mathrm{j}_{\mathrm{p}}{ }^{2}=.12\right)$, and type of sequence and order $\left(\mathrm{F}_{1.778,149.353}=13.28, \mathrm{p}<.001, \mathrm{j}_{\mathrm{p}}{ }^{2}=.14\right)$.

Further ANOVAs restricted to the left and right DMS (Table 2) revealed that: 1) non-dominant ambiguous and unambiguous sequences showed larger activation than dominant sequences in bilateral anterior and posterior DMS; nevertheless, in the left anterior and posterior DMS ambiguous non-dominant sequences displayed larger activation than unambiguous non-dominant sequences; 2) while bilateral posterior DMS displayed no differences between ambiguous non-dominant sequences and incongruent sequences, larger activation for A21 was evident in anterior DMS; moreover, only ambiguous sequences (compared to incongruent) showed larger activation for order 2-1 than order 1-2 in bilateral anterior and posterior DMS.

Thus, the parameter estimates analysis indicated larger activation in the DMS for less likely non-dominant sequences (both, ambiguous and unambiguous) compared to most common dominant sequences; in addition, the results indicated larger activation in left DMS for less common sequences with uncertain contextual evidence (A21) compared to uncommon sequences that provided precise contextual evidence (U221). Further, ambiguous nondominant and incongruent conditions, which both deviate from 
participants' expectations, displayed larger activation in posterior DMS; larger activation in anterior DMS was observed for the condition with a less common correct alternative (i.e. ambiguous non-dominant) compared to the outright incorrect condition (i.e. incongruent condition). Importantly, these results replicate our previous studies (Mestres-Misse et al., 2012, 2014a).

\section{Regression analysis}

The results of the whole-brain regression analysis revealed a significant cluster in the interthalamic nuclei (height threshold: $\mathrm{p}=.0005 ;$ peak voxel: $-3, \quad-11,0 ; \mathrm{t}=6.53 ; \mathrm{kE}=157$; pFWE $<.001$ ), which positively correlated with the difference between A21 sequences' response times minus U221 sequences' response times (Inline Supplementary Fig. S1). The cluster included bilateral central medial nucleus (CeM: $-3,-11,0 ; 3,-6$, $-2, \mathrm{t}=5.47)$ and right centromedian-parafascicular complex (CM-Pf: $3,-17,0, \mathrm{t}=5.25 ; 6,-20,-2, \mathrm{t}=5.08$ ).

The results of the whole-brain regression analysis revealed a significant cluster in the interthalamic nuclei (height threshold: $\mathrm{p}=.0005 ;$ peak voxel: $\quad-3, \quad-11,0 ; \mathrm{t}=6.53 ; \mathrm{kE}=157$; pFWE $<.001$ ), which positively correlated with the difference between A21 sequences' response times minus U221 sequences' response times (Inline Supplementary Fig. S1). The cluster included bilateral central medial nucleus (CeM: $-3,-11,0 ; 3,-6$, $-2, \mathrm{t}=5.47)$ and right centromedian-parafascicular complex (CM-Pf: $3,-17,0, \mathrm{t}=5.25 ; 6,-20,-2, \mathrm{t}=5.08$ ).

Inline Supplementary Fig. S1 can be found online at http://dx. doi.org/10.1016/j.neuroimage.2016.05.069.

The regression analysis indicated that the longer the processing times for ambiguous non-dominant sequences, the larger the activation in interthalamic nuclei.

\section{Functional connectivity analysis}

gPPI identified brain regions that were functionally connected to the left and right CM-Pf complex, and left and right anterior and posterior DMS (Inline Supplementary Fig. S2, Table 3). The left CM-Pf complex revealed significant functional connectivity with left anterior DMS. While the left anterior DMS showed increased functional connectivity with the left mediodorsal (MD) and VA nuclei of the thalamus, the right anterior DMS displayed increased functional connectivity with the right anterior dorsolateral prefrontal cortex, left angular gyrus, and the left anteroventral and ventral lateral posterior thalamic nuclei. Left posterior DMS showed increased functional connectivity with bilateral precuneus and cuneus. Right posterior DMS revealed increased functional connectivity with left posterior DMS, left VA and MD thalamic nuclei, left anterior DLS, and left frontal operculum/anterior insula extending to the inferior frontal gyrus (opercular part), claustrum and insula.

gPPI identified brain regions that were functionally connected to the left and right CM-Pf complex, and left and right anterior and posterior DMS (Inline Supplementary Fig. S2, Table 3). The left CM-Pf complex revealed significant functional connectivity with left anterior DMS. While the left anterior DMS showed increased functional connectivity with the left mediodorsal (MD) and VA nuclei of the thalamus, the right anterior DMS displayed increased functional connectivity with the right anterior dorsolateral prefrontal cortex, left angular gyrus, and the left anteroventral and ventral lateral posterior thalamic nuclei. Left posterior DMS showed increased functional connectivity with bilateral precuneus and cuneus. Right posterior DMS revealed increased functional connectivity with left posterior DMS, left VA and MD thalamic nuclei, left anterior DLS, and left frontal operculum/anterior insula extending to the inferior frontal gyrus (opercular part), claustrum and insula.

Inline Supplementary Fig. S2 can be found online at http://dx. doi.org/10.1016/j.neuroimage.2016.05.069.

The functional connectivity analysis indicated functional coupling between specific thalamic nuclei (i.e. CM-Pf complex, MD, VA) and the DMS, as well as between the DMS and frontal, parietal and insular regions during A21 processing. Remarkably, anterior DMS was functionally connected to anterior dorsolateral prefrontal cortex, which replicates and further demonstrates our proposal of an anterior-posterior gradient of cognitive control in the DMS, equivalent to the well-known prefrontal cortex gradient.

\section{Subcortical results summary}

To summarize the results on subcortical regions, larger activation was evident for ambiguous and non-dominant 2-1 order conditions in the DMS, thalamus, and RN. In the DLS, incongruent sequences showed larger activation compared to ambiguous sequences (Fig. 2).

Specifically, in the right DMS larger activation was observed for both congruent non-dominant orders (A21 and U221) compared to dominant orders (A12 and U112); while this was also the case for the left DMS, non-dominant ambiguous sequences showed larger activation than non-dominant unambiguous sequences in left DMS (Fig. 4 and Table 2). In posterior DMS incongruent conditions showed larger activation than in anterior DMS, as well as no significant differences compared with ambiguous sequences (Fig. 4 and Table 2). Additionally, the anterior DMS showed increased functional connectivity with the left thalamus (left: MD and VA nuclei), left angular gyrus (only right) and right anterior prefrontal cortex (only right) during A21 processing (Table 3 and Inline Supplementary Fig. S2). The right posterior DMS displayed increased functional connectivity with the left thalamus (MD and VA nuclei), left posterior DMS, left anterior DLS and the left frontal operculum/anterior insula, and the left posterior DMS showed increased coupling with the cuneus and precuneus during A21 processing (Table 3 and Inline Supplementary Fig. S2).

Specifically, in the right DMS larger activation was observed for both congruent non-dominant orders (A21 and U221) compared to dominant orders (A12 and U112); while this was also the case for the left DMS, non-dominant ambiguous sequences showed larger activation than non-dominant unambiguous sequences in left DMS (Fig. 4 and Table 2). In posterior DMS incongruent conditions showed larger activation than in anterior DMS, as well as no significant differences compared with ambiguous sequences (Fig. 4 and Table 2). Additionally, the anterior DMS showed increased functional connectivity with the left thalamus (left: MD and VA nuclei), left angular gyrus (only right) and right anterior prefrontal cortex (only right) during A21 processing (Table 3 and Inline Supplementary Fig. S2). The right posterior DMS displayed increased functional connectivity with the left thalamus (MD and VA nuclei), left posterior DMS, left anterior DLS and the left frontal operculum/anterior insula, and the left posterior DMS showed increased coupling with the cuneus and precuneus during A21 processing (Table 3 and Inline Supplementary Fig. S2).

The RN displayed larger activation for ambiguous conditions compared to unambiguous and incongruent conditions (Fig. 2); significant results, but uncorrected for multiple comparisons, comparing A21 > U221 (height threshold: $\mathrm{p}=.001$; peak voxel: $5,-21,-8 ; \mathrm{t}=5.05 ; \mathrm{kE}=24 ;$ Puncorr $=.021)$ and $\mathrm{A} 21>\mathrm{I} 212$ (height threshold: $\mathrm{p}=.001$; peak voxel: $-3,-23,-6 ; \mathrm{t}=5.00$; $\mathrm{kE}=21$; Puncorr $=.036$ ) hint that this effect may be caused primarily by larger activation for non-dominant ambiguous conditions in this region (Fig. 4).

The CM-Pf complex showed larger activation for ambiguous sequences compared to incongruent sequences (Fig. 2). Similarly to the DMS and RN, this effect seems to be due to a larger activation for ambiguous non-dominant sequences (Fig. 4). This was suggested by the results from the functional connectivity analysis where the left CM-Pf showed an increased functional coupling with the left anterior DMS during A21 processing (Table 3 and Inline Supplementary Fig. S2), an increase in BOLD response in 

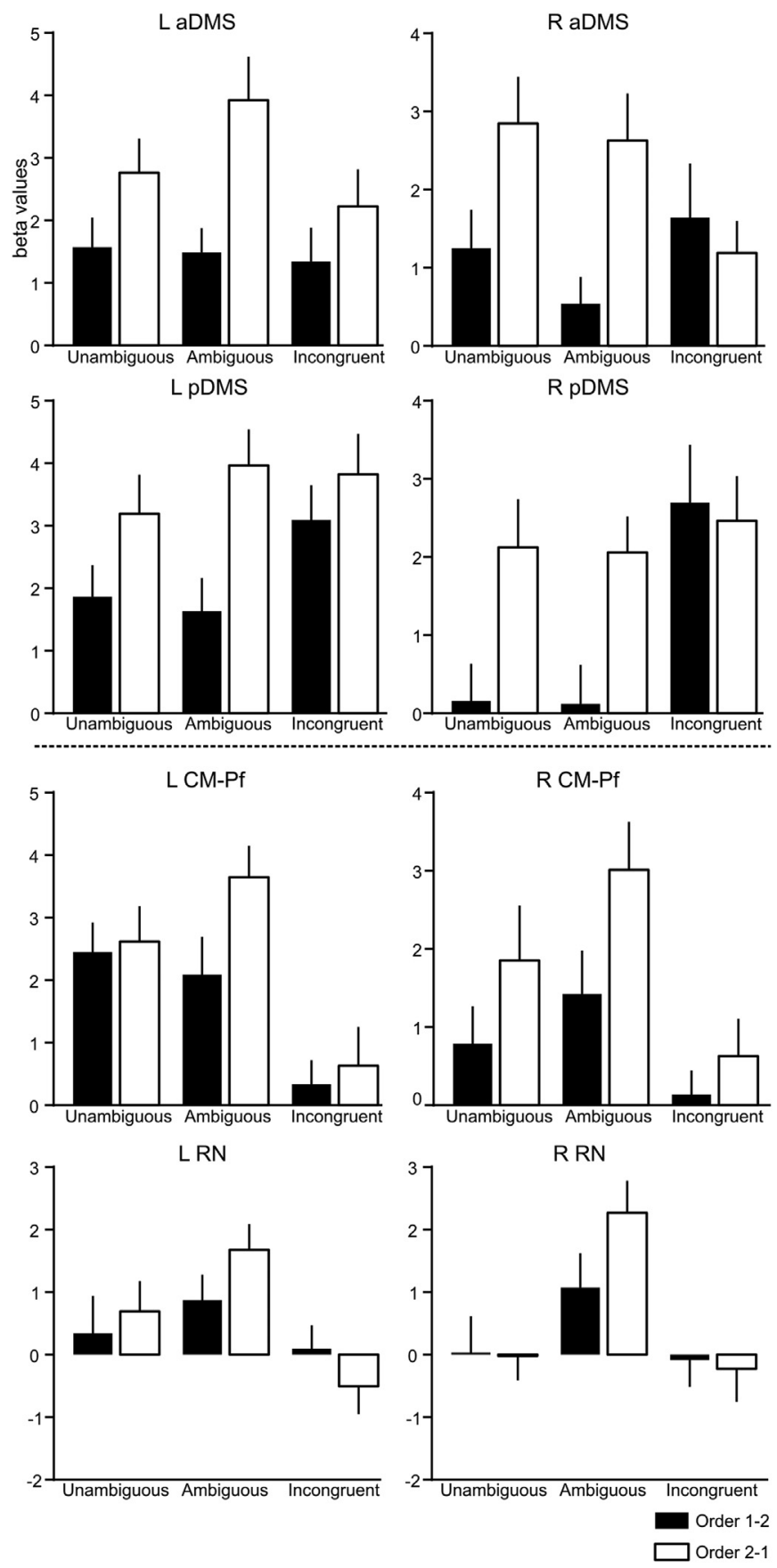

Fig. 4. Parameter estimates analysis. Group-average beta values (SEM error bars) for each condition in bilateral anterior (a) and posterior (p) dorsomedial striatum (DMS), centromedian-parafascicular (CM-Pf) complex and red nucleus (RN).

right CM-Pf as A21 sequences processing effort increased (measured as the difference between A21 and U221 RT) (Inline Supplementary Fig. S1), and a significant but uncorrected for multiple comparisons larger activation in the right CM-Pf for A21 compared to A12 (height threshold: $\mathrm{p}=.001$; peak voxel: $5,-21,-2$; $\mathrm{t}=5.54 ; \mathrm{kE}=20$; Puncorr $=.042$ ).

The CM-Pf complex showed larger activation for ambiguous sequences compared to incongruent sequences (Fig. 2). Similarly to the DMS and RN, this effect seems to be due to a larger activation for ambiguous non-dominant sequences (Fig. 4). This was suggested by the results from the functional connectivity analysis where the left CM-Pf showed an increased functional coupling with the left anterior DMS during A21 processing (Table 3 and Inline Supplementary Fig.
S2), an increase in BOLD response in right CM-Pf as A21 sequences processing effort increased (measured as the difference between A21 and U221 RT) (Inline Supplementary Fig. S1), and a significant but uncorrected for multiple comparisons larger activation in the right CM-Pf for A21 compared to A12 (height threshold: $\mathrm{p}=.001$; peak voxel: $5,-21,-2 ; \mathrm{t}=5.54 ; \mathrm{kE}=20$; Puncorr $=.042$ ).

Lastly, larger VA thalamic nucleus activation was found for the non-dominant 2-1 order compared to the dominant 1-2 order in both congruent (ambiguous and unambiguous) conditions (Fig. 2).

Collectively, larger activation was observed for the less likely non-dominant sequences in DMS and VA; in addition, only in the left DMS ambiguous non-dominant sequences displayed larger activation than unambiguous non-dominant sequences. Moreover, sequences which provided uncertain contextual evidence largely engaged the RN and the CM-Pf complex compared to the other sequences. Furthermore, while both sequences that violated participants' expectations activated the posterior DMS, only less likely, but correct sequences (A21) also showed larger activation in anterior DMS. Finally, the functional connectivity results support functional coupling between DMS and specific thalamic nuclei, as well as between DMS and frontal, parietal and insular regions, being particularly remarkable the functional connectivity between anterior DMS and anterior dorsolateral prefrontal cortex.

\section{Discussion}

We live in a world full of uncertainty. In order to deal with this uncertainty our brain has to learn the causal structure of the world and compute estimates of probable causes and outcomes by combining prior knowledge with currently available information. However, although being prepared for the most likely outcome is advantageous, sometimes a less likely outcome occurs. This unexpected event may be an uncommon one for which its probability of occurrence should be considered for future inference, or a one-time mistake. The present research investigated the contribution of subcortical structures to the process of determining estimates of probable outcomes, and their role in regulating uncertainty and deviations from expectation. Specifically, this was studied in situations where a most probable outcome is selected, due to contextual uncertainty and learned prior biases; however, the arrival of explicit evidence creates a conflict as the expected outcome does not match the actual outcome. Precisely on those occasions when participants process ambiguous sequences, which do not provide precise contextual information, they learned to expect the dominant outcome; but when the last-item turns out to be a white circle indicating the non-dominant order, a conflict arises with the ongoing dominant process (order 1-2, 1-item +2 -item). In this situation, cognitive control mechanisms are required to override this current strategy and to select and apply the less common, but appropriate one (order 2-1, 2-item - 1-item). The behavioral and imaging results are congruent in that the ambiguous $2-1$ condition is the one that requires the greatest processing resources. To a lesser extent, the unambiguous non-dominant 2-1 order and incongruent conditions do also pose higher processing demands than the dominant order 1-2.

In line with our previous reports (Mestres-Misse et al., 2012, 2014a), the present study reveals a similar pattern of activation in DMS. Generally, DMS displays larger activation for the nondominant 2-1 order; however, while in the right anterior and posterior DMS both the unambiguous and ambiguous non-dominant order displays larger activation than the dominant order 1-2, in left anterior and posterior DMS this activation was largest for non-dominant ambiguous sequences. Furthermore, incongruent sequences showed increased activation only in posterior DMS regions. These results are in accord with previous reports, namely, less expected stimuli, uncertain stimuli, and incongruent stimuli elicit increased striatal activation, but only those stimuli with a 
Table 2

Mixed ANOVAs for the left and right DMS

\begin{tabular}{llllllllll}
\hline L DMS & $\mathrm{A} / \mathrm{U}$ & & $\mathrm{A} / \mathrm{I}$ & & $\mathrm{R}$ DMS & $\mathrm{A} / \mathrm{U}$ & & $\mathrm{A} / \mathrm{I}$ & \\
& $\mathrm{F}$ & $\mathrm{j}_{\mathrm{p}}{ }^{2}$ & $\mathrm{~F}$ & $\mathrm{j}_{\mathrm{p}}{ }^{2}$ & & $\mathrm{~F}$ & $\mathrm{j}_{\mathrm{p}}{ }^{2}$ & $\mathrm{~F}$ & $\mathrm{j}_{\mathrm{p}}{ }^{2}$ \\
\hline Type & $\mathrm{ns}$ & .07 & $\mathrm{~ns}$ & .01 & Type & $\mathrm{ns}$ & .03 & $\mathrm{~ns}$ & .07 \\
Order & $58.68^{* * *}$ & .58 & $37.69^{* * *}$ & .47 & Order & $43.99^{* * *}$ & .51 & $10.62^{* *}$ & .20 \\
Ant. & $\mathrm{ns}$ & .00 & $\mathrm{~ns}$ & .04 & Ant. & $\mathrm{ns}$ & .03 & $\mathrm{~ns}$ & .01 \\
TxA & $\mathrm{ns}$ & .01 & $9.62^{* *}$ & .19 & TxA & $\mathrm{ns}$ & .02 & $4.87^{*}$ & .10 \\
OxA & $\mathrm{ns}$ & .00 & $\mathrm{~ns}$ & .00 & OxA & $\mathrm{ns}$ & .00 & $\mathrm{~ns}$ & .00 \\
TxO & $6.77^{*}$ & .14 & $9.17^{* *}$ & .18 & TxO & $\mathrm{ns}$ & .01 & $14.21^{* * *}$ & .25 \\
TxOxA & $\mathrm{ns}$ & .00 & $\mathrm{~ns}$ & .00 & TxOxA & $\mathrm{ns}$ & .01 & $\mathrm{~ns}$ & .00 \\
\hline
\end{tabular}

Ambiguous (A); Unambiguous (U); Incongruent (I); Type of sequence $(\mathrm{T})$; Order (O); Anteriority (Ant., A). L: left; R: right; DMS: dorsomedial striatum. Degrees of freedom: $1,42 .{ }^{*} \mathrm{p}<.05 ;{ }^{* *} \mathrm{p}<.01 ;{ }^{* * *} \mathrm{p}<.001$; ns: not significant.

Table 3

Functional connectivity during A21 processing for each thalamic and striatal seed region.

\begin{tabular}{|c|c|c|c|c|c|c|}
\hline \multirow{2}{*}{$\begin{array}{l}\text { Seed region } \\
\text { Anatomical area }\end{array}$} & \multicolumn{6}{|c|}{ Stereotaxic coordinates } \\
\hline & $\mathrm{x}$ & $\mathrm{y}$ & $\mathrm{z}$ & $\mathrm{t}$ & $\mathrm{kE}$ & $\mathrm{P}_{\mathrm{FWE}}$ \\
\hline \multicolumn{7}{|l|}{ L CM-Pf complex } \\
\hline$\overline{\mathrm{L} \text { anterior dorsomedial striatum }}$ & -11 & 12 & 5 & 6.14 & 130 & $<.001$ \\
\hline R CM-Pf complex & \multicolumn{6}{|c|}{ No significant regions } \\
\hline \multicolumn{7}{|l|}{ L anterior dorsomedial striatum } \\
\hline$\overline{\mathrm{L} \text { mediodorsal nucleus }}$ & -8 & -20 & 11 & 5.17 & 175 & $<.001$ \\
\hline L ventral anterior nucleus & -11 & -11 & 8 & 4.77 & & \\
\hline \multicolumn{7}{|l|}{$R$ anterior dorsomedial striatum } \\
\hline $\mathrm{R}$ anterior middle frontal $\mathrm{g}$. & 53 & 47 & 8 & 5.61 & 102 & .003 \\
\hline $\mathrm{L}$ angular $\mathrm{g}$. & -38 & -69 & 36 & 5.45 & 95 & .005 \\
\hline $\mathrm{L}$ anteroventral nucleus & -5 & -14 & 18 & 5.10 & 65 & .046 \\
\hline L ventral lateral posterior nucleus & -12 & -12 & 12 & 4.74 & & \\
\hline \multicolumn{7}{|l|}{ L posterior dorsomedial striatum } \\
\hline$\overline{\mathrm{R} \text { precuneus }}$ & 20 & -56 & 18 & 6.96 & 194 & $<.001$ \\
\hline L cuneus & -3 & -78 & 2 & 6.79 & 1057 & $<.001$ \\
\hline $\mathrm{R}$ precuneus & 2 & -51 & 18 & 5.78 & 133 & .001 \\
\hline L precuneus & -11 & -69 & 23 & 5.12 & 153 & $<.001$ \\
\hline \multicolumn{7}{|l|}{$R$ posterior dorsomedial striatum } \\
\hline$\overline{\mathrm{L} \text { posterior dorsomedial striatum }}$ & -14 & 6 & 15 & 7.17 & 119 & .001 \\
\hline L ventral anterior nucleus & -11 & -11 & 8 & 6.99 & 202 & $<.001$ \\
\hline L mediodorsal nucleus & -5 & -17 & 8 & 5.04 & & \\
\hline L anterior dorsolateral striatum & -23 & 12 & 2 & 5.58 & 186 & $<.001$ \\
\hline L frontal operculum/anterior insula & -41 & 23 & 0 & 5.11 & 99 & .004 \\
\hline
\end{tabular}

Local maxima for the functional connectivity with the different defined seed regions (underlined) assessing increased coupling during the A21 condition. kE: cluster size, $\mathrm{mm}^{3}$; FWE, family-wise error; L, left; R, right; g: gyrus; CM-Pf: centromedian-parafascicular.

correct, less dominant alternative also engage more anterior parts of the DMS (Mestres-Misse et al., 2012, 2014a). Furthermore, and in contrast to our two previous studies, the current study also showed DLS activation. Interestingly, larger activation in DLS was restricted to incongruent sequences. This contrasting pattern of activation within the dorsal striatum may reflect two different levels of overriding a dominant behavior with an alternative one, that is, a cognitive set-shift in DMS and a response set-shift in DLS. Activation in DLS may reflect response updating; as participants automatically start calculating the value of the sequence before the last-item, an advanced predisposed 'yes, sequence is correct' response may be prepared, which would need to be counteracted in incongruent trials. This is consistent with studies employing a card-sorting task, which showed increased activation in DMS during the planning of a cognitive (rule) set-shift and increased DLS activation during the implementation of the appropriate response according to the new rule (Monchi et al., 2001, 2006; Nagano-Saito et al., 2008; Simard et al., 2011). Additionally, the observed activation is congruent with striatal afferents from cortex whereby lateral prefrontal cortex projects predominantly to DMS and premotor and motor cortices project to DLS (Kotz et al., 2013; Di Martino et al., 2008; Draganski et al., 2008; Haber, 2003; Parent and Hazrati, 1995). Note also that owing to the topography of striatal afferents (Haber, 2003; Parent and Hazrati, 1995), the most dorsal and caudal part of the DMS (body of the caudate) also receive premotor cortex projections, which is consistent with the observed increased activation for incongruent sequences in this region. Consistently with these results, the striatum is shown to have a particular role in overriding dominant and/or pre-biased responses, choosing appropriate responses among competing alternatives and suppressing unwanted ones, and switching from a planned or automatic response to an alternative or controlled one in light of a sudden, unexpected change (e.g. Cameron et al., 2009; Cools et al., 2006; den Ouden et al., 2010; Hikosaka and Isoda, 2010; Mestres-Misse et al., 2012, 2014a; Redgrave et al., 1999; Schiffer et al., 2015). Consequently, the present results support the decisive function of the striatum in behavioral and cognitive control, flexibility, and adjustment. Importantly, these results, together with previous studies, demonstrate that, on the one hand, functional and topographic precision within the basal ganglia is possible, and thus, function can be precisely localized in particular subregions within the striatum (i.e. ventral, dorsomedial, and dorsolateral striatum). Future studies should therefore ascribe and discuss striatal function accordingly, avoiding ascribing a particular function to the whole structure without considering established striatal topographic and functional differences. On the other hand, our studies show that the involvement of the basal ganglia in prediction errors, probability manipulations, violation of expectancy, is independent of explicit value and reward, and reflects a difference between value-based and non-value based processes indicated by topographical differentiation within the striatum (ventral striatum vs. dorsomedial striatum).

The DMS was not the only subcortical structure that showed increased activation for non-dominant ambiguous sequences. In bilateral RN, ambiguous sequences led to increased activation when compared to the other conditions. This is probably the result of increased activation for non-dominant ambiguous sequences. Remarkably, the human RN has been suggested to be involved in higher cognitive functions (Habas and Cabanis, 2007; Nioche et al., 2009). This nucleus receives afferents from associative cortical regions, although its main source of afferents is the dentate nucleus, and the RN projects mainly to the inferior olive (Habas and Cabanis, 2007; Nioche et al., 2009; Schmahmann and Sherman, 1998). Connections between the RN and basal ganglia have also been suggested (Habas and Cabanis, 2007). Even though the exact functionality of the human RN is not yet clear, the rubral network is suggested to be involved in the detection of discrepancies between expected and unexpected outcomes (Habas et al., 2010; Nioche et al., 2009). This functional role ascribed to the human RN matches well with the current results and this serve to endorse the involvement of the human RN in higher cognitive function.

Similarly, the bilateral CM-Pf complex displayed increased activation for ambiguous sequences; again, mainly as a result of higher activation for non-dominant ambiguous sequences. Through the CM-Pf complex the thalamus sends functionally and topographically organized projections to the striatum (Haber and McFarland, 2001; Haber and Calzavara, 2009; Smith et al., 2004, 2014). This thalamo-striatal projection exerts direct modulation of the striatum, so that thalamic regions not only relay information from the basal ganglia to cortex, but the same regions project back to the striatum, providing direct feedback (Haber and McFarland, 2001). CM-Pf neurons are characterized as having multimodal properties and being sensitive to salient and attention-demanding events (Smith et al., 2004, 2009). Notably, neurons in the monkey CM-Pf complex have been shown to respond to unpredictable or unexpected stimuli (Matsumoto et al., 2001; 
Minamimoto et al., 2005; Minamimoto and Kimura, 2002). These authors propose that the CM-Pf complex receives, via the basal ganglia, information regarding the expected, biased response, whereas cortico-thalamic connections provide information about actual events. Hence the CM-Pf complex appears to track discrepancies between both sets of information (Kimura et al., 2004; Minamimoto et al., 2005, 2009; Smith et al., 2011). Furthermore, the CM-Pf complex is crucially involved not only in detecting events that are different from expectation, but also in overcoming response biases (Minamimoto et al., 2009, 2014; Smith et al., 2011). When a discrepancy between the internal bias and external evidence is detected by the CM-Pf complex, it discharges a signal, which triggers in the striatum an override of the dominant response, and the selection of an appropriate alternative (Minamimoto et al., 2009, 2014). The basal ganglia and CM-Pf complex may thus work together in coordinating habitual and controlled processing, facilitating cognitive and behavioral flexibility (Brown et al., 2010) in so doing. Interestingly, in the present study, the pattern of activation observed in the CM-Pf complex resembled that from the anterior DMS. Furthermore, these two regions demonstrated functional coupling. We could venture to speculate that the differences observed between anterior and posterior DMS may reflect CM-Pf modulation of striatal activity after a discrepancy between presumed and actual outcomes is detected in the CM-Pf complex. Additionally, the role of CM-Pf complex in tracking differences between expected and actual outcomes is further supported by our regression analysis, which revealed a positive relation between increased RT for A21 and activity in this region. Nevertheless, this activation was not localized but extended to other thalamic nuclei.

Furthermore, the VA thalamic nucleus revealed larger activation for congruent (unambiguous and ambiguous) non-dominant sequences compared with dominant ones. The ventral anterior and ventral lateral $(\mathrm{VA} / \mathrm{VL})$ thalamic nuclei are the major targets of basal ganglia output and relay this output to cortex. At the same time, VA/VL also provide input to the dorsal striatum, which has been suggested to supply the striatum with indirect cortical information (McFarland and Haber, 2001). Moreover, VA has robust connections with prefrontal and anterior cingulate cortices (McFarland and Haber, 2002; Xiao and Barbas, 2004). Altogether this is consistent with the observed increased activation of this nucleus for non-dominant orders and the increased functional connectivity between the dorsomedial striatum and thalamus.

Lastly, it should be noted that because the focus of this study is on subcortical structures, cortical activations are not discussed in detail. The role of the prefrontal cortex in executive functions has been extensively studied (Badre, 2008; Badre and D'Esposito, 2009; Botvinick, 2008; Duncan and Owen, 2000; Koechlin et al., 2003; Miller and Cohen, 2001; Petrides, 2005) and cortico-striatial connections are largely known (Kemp and Powell, 1970; Selemon and Goldman-Rakic, 1985; Yeterian and Pandya, 1991). The present study revealed several lateral frontal cortex as well as parietal activations consistent with the central executive network next to anterior insula/frontal operculum, medial frontal, and anterior cingulate activations corresponding to the salience network (Seeley et al., 2007). Collectively these regions have been suggested to constitute a multiple-demand system commonly activated across numerous complex cognitive tasks and related to human intelligence (Duncan, 2010, 2013; Fedorenko et al., 2013). Here reflecting, particularly when expectations were violated, higher-level cognitive processes such as detection of unexpected outcomes, performance monitoring, attentional control, inhibitory control, setshift, cognitive flexibility, and working memory (including retrieval and manipulation of information). Furthermore, the anterior dorsomedial striatum showed increased functional coupling with the anterior lateral frontal cortex and angular gyrus. This functional connectivity between anterior DMS and anterior dorsolateral prefrontal cortex further supports our previous suggestion of an anterior-posterior gradient of cognitive control in the DMS (Mestres-Misse et al., 2012, 2014a), mimicking a prefrontal cortex gradient (Badre, 2008; Badre and D'Esposito, 2009; Koechlin et al., 2003). Therefore, we propose that the coordination of several cortical regions, i.e. lateral and medial prefrontal, insular and parietal cortices, is paramount to probabilistic inferential learning, dealing with uncertainty and general flexible cognitive control, and that the basal ganglia and thalamus, owing to their connections, are at the core of the modulation and control of these cognitive processes.

\section{Conclusions}

To conclude, we have further proved the crucial function of subcortical structures in regulating uncertainty as well as the balance between automatic and controlled processing; this is particularly critical during learning as deviations from expectations prompt to boost learning. Accordingly, the results affirm the role of the striatum in overriding a dominant response when external evidence triggers its inadequacy as previously shown in highly proficient behavior (i.e. language) (Mestres-Misse et al., 2012, 2014a). Furthermore, the results reveal that during less wellestablished behavior, which benefits from expectancy violations as it provides an opportunity to update beliefs, other subcortical regions also contribute to cognitive control processes. Furthermore, the present results suggest that cognitive and behavioral flexibility requires the cooperation and coordination of cortical and subcortical regions, which is achieved via various complex and interacting cortico-subcortical circuities.

\section{Acknowledgments}

This work was supported by a Beatriu de Pinós Postdoctoral Grant (BP-DGR, 2009 BP-A 00025) from the Government of Catalonia (Generalitat de Catalunya) to AMM and by a Canadian subaward (CHIR 62667) to SAK. We are grateful to Iris Nikola Knierim for her help with programming the experiment and Andreas Schäfer for his help with the fieldmap correction.

\section{Appendix A. Supplementary data}

Supplementary data to this article can be found online at http://dx.doi.org/10.1016/j.neuroimage.2016.05.069.

\section{References}

Badre, D., 2008. Cognitive control, hierarchy, and the rostro-caudal organization of the frontal lobes. Trends Cogn. Sci. 12, 193-200.

Badre, D., D'Esposito, M., 2009. Is the rostro-caudal axis of the frontal lobe hierarchical? Nat. Rev. Neurosci. 10, 659-669.

Behrens, T.E., Woolrich, M.W., Walton, M.E., Rushworth, M.F., 2007. Learning the value of information in an uncertain world. Nat. Neurosci. 10, 1214-1221.

Botvinick, M.M., 2008. Hierarchical models of behavior and prefrontal function. Trends Cogn. Sci. 12, 201-208.

Brown, H.D., Baker, P.M., Ragozzino, M.E., 2010. The parafascicular thalamic nucleus concomitantly influences behavioral flexibility and dorsomedial striatal acetylcholine output in rats. J. Neurosci. 30, 14390-14398.

Cameron, I.G., Coe, B.C., Watanabe, M., Stroman, P.W., Munoz, D.P., 2009. Role of the basal ganglia in switching a planned response. Eur. J. Neurosci. 29, 2413-2425. Cools, R., Ivry, R.B., D'Esposito, M., 2006. The human striatum is necessary for responding to changes in stimulus relevance. J. Cogn. Neurosci. 18, 1973-1983.

den Ouden, H.E., Daunizeau, J., Roiser, J., Friston, K.J., Stephan, K.E., 2010. Striatal prediction error modulates cortical coupling. J. Neurosci. 30, 3210-3219.

Di Martino, A., Scheres, A., Margulies, D.S., Kelly, A.M., Uddin, L.Q., Shehzad, Z Biswal, B., Walters, J.R. Castellanos, FX Milham, M.P, 2008. Functional connectivity of human striatum: a resting state FMRI study. Cereb. Cortex 18, 2735-2747.

Draganski, B., Kherif, F., Kloppel, S., Cook, P.A., Alexander, D.C., Parker, G.J., Deichmann, R., Ashburner, J., Frackowiak, R.S., 2008. Evidence for segregated and 
integrative connectivity patterns in the human basal ganglia. J. Neurosci. 28, 7143-7152.

Duncan, J., 2010. The multiple-demand (MD) system of the primate brain: mental programs for intelligent behaviour. Trends Cogn. Sci. 14, 172-179.

Duncan, J., 2013. The structure of cognition: attentional episodes in mind and brain. Neuron 80, 35-50.

Duncan, J., Owen, A.M., 2000. Common regions of the human frontal lobe recruited by diverse cognitive demands. Trends Neurosci. 23, 475-483.

Edelstein, W.A., Glover, G.H., Hardy, C.J., Redington, R.W., 1986. The intrinsic signalto-noise ratio in NMR imaging. Magn. Reson. Med. 3, 604-618.

Fedorenko, E., Duncan, J., Kanwisher, N., 2013. Broad domain generality in focal regions of frontal and parietal cortex. Proc. Natl. Acad. Sci. U. S. A. 110, $16616-16621$.

Fiser, J., Berkes, P., Orban, G., Lengyel, M., 2010. Statistically optimal perception and learning: from behavior to neural representations. Trends Cogn. Sci. 14, 119-130.

Friston, K.J., Holmes, A.P., Worsley, K.J., Poline, J.B., Frith, C.D., Frackowiak, R.S., 1995. Statistical parametric maps in functional imaging: a general linear approach. Hum. Brain Mapp. 2, 189-210.

Habas, C., Cabanis, E.A., 2007. Cortical projection to the human red nucleus: complementary results with probabilistic tractography at 3 T. Neuroradiology 49, 777-784.

Habas, C., Guillevin, R., Abanou, A., 2010. In vivo structural and functional imaging of the human rubral and inferior olivary nuclei: a mini-review. Cerebellum 9, $167-173$.

Haber, S.N., 2003. The primate basal ganglia: parallel and integrative networks. J. Chem. Neuroanat. 26, 317-330.

Haber, S.N., Calzavara, R., 2009. The cortico-basal ganglia integrative network: the role of the thalamus. Brain Res. Bull. 78, 69-74.

Haber, S., McFarland, N.R., 2001. The place of the thalamus in frontal cortical-basal ganglia circuits. Neuroscientist 7, 315-324.

Hikosaka, O., Isoda, M., 2010. Switching from automatic to controlled behavior: cortico-basal ganglia mechanisms. Trends Cogn. Sci. 14, 154-161.

Kemp, J.M., Powell, T.P., 1970. The cortico-striate projection in the monkey. Brain 93, 525-546.

Kimura, M., Minamimoto, T., Matsumoto, N., Hori, Y., 2004. Monitoring and switching of cortico-basal ganglia loop functions by the thalamo-striatal system. Neurosci. Res. 48, 355-360.

Knill, D.C., Pouget, A., 2004. The Bayesian brain: the role of uncertainty in neural coding and computation. Trends Neurosci. 27, 712-719.

Koechlin, E., Ody, C., Kouneiher, F., 2003. The architecture of cognitive control in the human prefrontal cortex. Science 302, 1181-1185.

Kotz, S.A., Anwander, A., Axer, H., Knosche, T.R., 2013. Beyond cytoarchitectonics: the internal and external connectivity structure of the caudate nucleus. PLoS One 8, e70141.

Mai, J.K., Paxinos, G., 2008. Atlas of the Human Brain. Academic Press.

Marques, J.P., Kober, T., Krueger, G., van der Zwaag, W., Van de Moortele, P.F., Gruetter, R., 2010. MP2RAGE, a self bias-field corrected sequence for improved segmentation and T1-mapping at high field. NeuroImage 49, 1271-1281.

Matsumoto, N., Minamimoto, T., Graybiel, A.M., Kimura, M., 2001. Neurons in the thalamic CM-Pf complex supply striatal neurons with information about behaviorally significant sensory events. J. Neurophysiol. 85, 960-976.

McFarland, N.R., Haber, S.N., 2001. Organization of thalamostriatal terminals from the ventral motor nuclei in the macaque. J. Comp. Neurol. 429, 321-336.

McFarland, N.R., Haber, S.N., 2002. Thalamic relay nuclei of the basal ganglia form both reciprocal and nonreciprocal cortical connections, linking multiple frontal cortical areas. J. Neurosci. 22, 8117-8132.

McLaren, D.G., Ries, M.L., Xu, G., Johnson, S.C., 2012. A generalized form of contextdependent psychophysiological interactions (gPPI): a comparison to standard approaches. Neurolmage 61, 1277-1286.

Mestres-Misse, A., Turner, R., Friederici, A.D., 2012. An anterior-posterior gradient of cognitive control within the dorsomedial striatum. NeuroImage 62, 41-47.

Mestres-Misse, A., Bazin, P.L., Trampel, R., Turner, R., Kotz, S.A., 2014a. Dorsomedial striatum involvement in regulating conflict between current and presumed outcomes. NeuroImage 98C, 159-167.

Mestres-Misse, A., Trampel, R., Turner, R., Kotz, S.A., 2014b. In favor of general probability distributions: lateral prefrontal and insular cortices respond to stimulus inherent, but irrelevant differences. Brain Struct. Funct.

Miller, E.K., Cohen, J.D., 2001. An integrative theory of prefrontal cortex function. Annu. Rev. Neurosci. 24, 167-202.

Minamimoto, T., Kimura, M., 2002. Participation of the thalamic CM-Pf complex in attentional orienting. J. Neurophysiol. 87, 3090-3101.

Minamimoto, T., Hori, Y., Kimura, M., 2005. Complementary process to response bias in the centromedian nucleus of the thalamus. Science 308, 1798-1801.

Minamimoto, T., Hori, Y., Kimura, M., 2009. Roles of the thalamic CM-PF complexbasal ganglia circuit in externally driven rebias of action. Brain Res. Bull. 78, 75-79.
Minamimoto, T., Hori, Y., Yamanaka, K., Kimura, M., 2014. Neural signal for counteracting pre-action bias in the centromedian thalamic nucleus. Front. Syst. Neurosci. 8, 3.

Monchi, O., Petrides, M., Petre, V., Worsley, K., Dagher, A., 2001. Wisconsin card sorting revisited: distinct neural circuits participating in different stages of the task identified by event-related functional magnetic resonance imaging. J. Neurosci. 21, 7733-7741.

Monchi, O., Petrides, M., Strafella, A.P., Worsley, K.J., Doyon, J., 2006. Functional role of the basal ganglia in the planning and execution of actions. Ann. Neurol. 59, 257-264.

Morel, A., 2007. Stereotactic Atlas of the Human Thalamus and Basal Ganglia. Informa Healthcare USA, Inc..

Mushtaq, F., Bland, A.R., Schaefer, A., 2011. Uncertainty and cognitive control. Front. Psychol. 2, 249.

Nagano-Saito, A., Leyton, M., Monchi, O., Goldberg, Y.K., He, Y., Dagher, A., 2008. Dopamine depletion impairs frontostriatal functional connectivity during a setshifting task. J. Neurosci. 28, 3697-3706.

Nioche, C., Cabanis, E.A., Habas, C., 2009. Functional connectivity of the human red nucleus in the brain resting state at 3T. AJNR Am. J. Neuroradiol. 30, 396-403.

O'Reilly, J.X., 2013. Making predictions in a changing world-inference, uncertainty, and learning. Front. Neurosci. 7, 105.

Parent, A., Hazrati, L.N., 1995. Functional anatomy of the basal ganglia. I. The cortico-basal ganglia-thalamo-cortical loop. Brain Res. Brain Res. Rev. 20, 91-127.

Penny, W., 2012. Bayesian models of brain and behaviour. ISRN Biomath. 2012.

Petrides, M., 2005. The Rostral-Caudal Axis of Cognitive Control within the Lateral Frontal Cortex. In: Dehaene, S., Duhamel, G.R., Hauser, G.R., Rizzolatti, G. (Eds.), From Monkey Brain to Human Brain. MIT Press, Cambridge, MA, pp. 293-314.

Pouget, A., Beck, J.M., Ma, W.J., Latham, P.E., 2013. Probabilistic brains: knowns and unknowns. Nat. Neurosci. 16, 1170-1178.

Pruessmann, K.P., Weiger, M., Scheidegger, M.B., Boesiger, P., 1999. SENSE: sensitivity encoding for fast MRI. Magn. Reson. Med. 42, 952-962.

Redgrave, P., Prescott, T.J., Gurney, K., 1999. The basal ganglia: a vertebrate solution to the selection problem? Neuroscience 89, 1009-1023.

Saffran, J.R., Aslin, R.N., Newport, E.L., 1996. Statistical learning by 8-month-old infants. Science 274, 1926-1928.

Schiffer, A.M., Waszak, F., Yeung, N., 2015. The role of prediction and outcomes in adaptive cognitive control. J. Physiol. Paris.

Schmahmann, J.D., Sherman, J.C., 1998. The cerebellar cognitive affective syndrome. Brain 121, 561-579.

Seeley, W.W., Menon, V., Schatzberg, A.F., Keller, J., Glover, G.H., Kenna, H., Reiss, A. L., Greicius, M.D., 2007. Dissociable intrinsic connectivity networks for salience processing and executive control. J. Neurosci. 27, 2349-2356.

Selemon, L.D., Goldman-Rakic, P.S., 1985. Longitudinal topography and interdigitation of corticostriatal projections in the rhesus monkey. J. Neurosci. 5, 776-794.

Simard, F., Joanette, Y., Petrides, M., Jubault, T., Madjar, C., Monchi, O., 2011. Frontostriatal contribution to lexical set-shifting. Cereb. Cortex 21, 1084-1093.

Smith, Y., Raju, D.V., Pare, J.F., Sidibe, M., 2004. The thalamostriatal system: a highly specific network of the basal ganglia circuitry. Trends Neurosci. 27, 520-527.

Smith, Y., Raju, D., Nanda, B., Pare, J.F., Galvan, A., Wichmann, T., 2009. The thalamostriatal systems: anatomical and functional organization in normal and parkinsonian states. Brain Res. Bull. 78, 60-68.

Smith, Y., Surmeier, D.J., Redgrave, P., Kimura, M., 2011. Thalamic contributions to basal ganglia-related behavioral switching and reinforcement. J. Neurosci. 31, 16102-16106.

Smith, Y., Galvan, A., Ellender, T.J., Doig, N., Villalba, R.M., Huerta-Ocampo, I., Wichmann, T., Bolam, J.P., 2014. The thalamostriatal system in normal and diseased states. Front. Syst. Neurosci. 8, 5.

Stahl, A.E., Feigenson, L., 2015. Cognitive development. Observing the unexpected enhances infants' learning and exploration. Science 348, 91-94.

Strotmann, B., Heidemann, R.M., Anwander, A., Weiss, M., Trampel, R., Villringer, A., Turner, R., 2014. High-resolution MRI and diffusion-weighted imaging of the human habenula at 7 tesla. J. Magn. Reson. Imaging 39, 1018-1026.

Summerfield, C., de Lange, F.P., 2014. Expectation in perceptual decision making: neural and computational mechanisms. Nat. Rev. Neurosci. 15, 745-756.

van der Zwaag, W., Schafer, A., Marques, J.P., Turner, R., Trampel, R., 2015. Recent applications of UHF-MRI in the study of human brain function and structure: a review. NMR Biomed.

Vilares, I., Kording, K., 2011. Bayesian models: the structure of the world, uncertainty, behavior, and the brain. Ann. N. Y. Acad. Sci. 1224, 22-39.

Xiao, D., Barbas, H., 2004. Circuits through prefrontal cortex, basal ganglia, and ventral anterior nucleus map pathways beyond motor control. Thalamus Relat. Syst. 2, 18.

Yeterian, E.H., Pandya, D.N., 1991. Prefrontostriatal connections in relation to cortical architectonic organization in rhesus monkeys. J. Comp. Neurol. 312, 43-67.

Yu, A.J., Dayan, P., 2005. Uncertainty, neuromodulation, and attention. Neuron 46, 681-692. 